\title{
Two-loop Fermionic Electroweak Corrections to the Effective Leptonic Weak Mixing Angle in the Standard Model *
}

\author{
M. Awramik a b, M. Czakon a c , A. Freitas d, G. Weiglein e \\ aDESY, Platanenallee 6, D-15738 Zeuthen, Germany \\ bInstitute of Nuclear Physics PAS, Radzikowskiego 152, PL-31342 Cracow, Poland \\ ${ }^{\mathrm{c}}$ Institute of Physics, University of Silesia, Uniwersytecka 4, PL-40007 Katowice, Poland \\ dTheoretical Physics Division, Fermilab, P. O. Box 500, Batavia, IL 60510, USA \\ eInstitute for Particle Physics Phenomenology, University of Durham, Durham DH1 3LE, UK
}

We give some details of the recently completed calculation of the full two-loop fermionic corrections to the effective leptonic weak mixing angle, $\sin ^{2} \theta_{\mathrm{eff}}^{\text {lept }}$. Among others, we describe the C ++ library DiaGen/IdSolver, which was used to reduce the two-loop light fermion vertex diagrams to linear combinations of master integrals with rational function coefficients.

\section{INTRODUCTION}

Two masses play at present an extremely important rôle among the parameters of the Standard Model. These are $M_{W}$ and $M_{H}$, the masses of the $\mathrm{W}$ and Higgs bosons respectively. As long as experiments will not reach a satisfactory level of precision for $M_{W}$ and will not be able to give a value to $M_{H}$ by direct observation, we are bound to seek indirect predictions for these observables based on theoretical calculations of suitably chosen processes. It is well known that $M_{W}$ can be predicted with the help of the precisely measured muon decay lifetime, while $M_{H}$ from the $\mathrm{Z}$ peak observables, most notably from the effective leptonic weak mixing angle, $\sin ^{2} \theta_{\text {eff }}^{\text {lept }}$.

The theoretical program for the $\mathrm{W}$ boson mass prediction has been completed with an error estimate of $4 \mathrm{MeV}$ [1], which is even below the expected precision of measurement at a future linear collider, not to mention the much closer Large

\footnotetext{
*Work supported in part by TMR, European Community Human Potential Programme under contracts HPRN-CT2002-00311 (EURIDICE), HPRN-CT-2000-00149 (Physics at Colliders), by Deutsche Forschungsgemeinschaft under contract SFB/TR 9-03, and by the Polish State Committee for Scientific Research (KBN) under contract No. 2P03B01025.
}

Hadron Collider (LHC), where the error should be of the order of $15 \mathrm{MeV}$. This achievement required among others the two-loop electroweak result finished with a calculation of the bosonic part [2], following a long study of the fermionic part $[4,5]$. To be complete we have to mention more than twenty years of various works starting from the original analysis [6], going through QCD corrections to the one-loop result $[7,8]$ and finally some three-loop leading contributions in the top quark mass [9].

In the case of the effective leptonic weak mixing angle, $\sin ^{2} \theta_{\text {eff }}^{\text {lept }}$, the situation is not so satisfactory. Most of the corrections listed above still apply, but a complete calculation at the two-loop level in the electroweak interactions does not exist, and only the $m_{t}^{4}$ and $m_{t}^{2}$ terms in the top quark mass expansion are available $[3,10]$. Since the current measurement has an impressive precision, $\sin ^{2} \theta_{\text {eff }}^{\text {lept }}=0.23150 \pm 0.00016[11]$, and prospects are to reach an absolute error of $10^{-5}$ at a future linear collider, it is still necessary to compute several contributions. Recently, we took a step in this direction by performing a complete calculation of the two-loop electroweak contributions generated by diagrams with one or two 
closed fermion loops [12]. In this contribution, some aspects of this calculation will be described. We will first present the method used for the top quark vertex diagrams, then that for the light fermion diagrams. Finally we will discuss some details of the $\mathrm{C}++$ library DiaGen/IdSolver [13] used for the decomposition of diagrams to combinations of master integrals.

\section{TOP QUARK CONTRIBUTIONS}

$\sin ^{2} \theta_{\text {eff }}^{\text {lept }}$ is defined through the form factors of the leptonic $\mathrm{Z}$ boson vertex. Namely, if the vertex is $i \bar{l} \gamma^{\mu}\left(g_{V}-g_{A} \gamma_{5}\right) l Z_{\mu}$, then

$$
\sin ^{2} \theta_{\mathrm{eff}}^{\mathrm{lept}}=\frac{1}{4}\left(1-\operatorname{Re}\left(\frac{g_{V}}{g_{A}}\right)\right) .
$$

The main complication in the calculation comes from the two-loop vertices that one has to evaluate. All other irreducible two-loop diagrams enter through renormalization constants and can be obtained from propagator diagrams at nonvanishing external momentum at worst. The algorithm to obtain numerical values of these has been completed in [14].

We decided to divide the fermionic two-loop vertices in two groups, one with top quark lines and one that has only light fermion lines. The contribution of the former is expressed through three massive parameters (we neglect the light fermion masses), $M_{W}, M_{Z}$ and $m_{t}$, and is thus a nontrivial function of two dimensionless variables $^{2}$. Although it is conceivable that the result could be expressed in some closed analytic form, this is absolutely not needed. It turns out that it is better to exploit the smallness of the ratio $M_{Z}^{2} / m_{t}^{2} \sim 1 / 4$, which corresponds to decoupling of the top quark. Obviously the construction of a high precision effective theory would be very difficult, since high order tensor operators would be needed. Instead, we simply performed a diagrammatic large mass expansion.

We checked that inclusion of terms of order $\left(M_{Z}^{2} / m_{t}^{2}\right)^{5}$ is sufficient to obtain an intrinsic precision of $10^{-7}$, which is by far enough for practical purposes. An example of a scalar diagram

${ }^{2}$ The fermionic vertex diagrams do not depend on the Higgs boson mass due to $C P$ conservation.

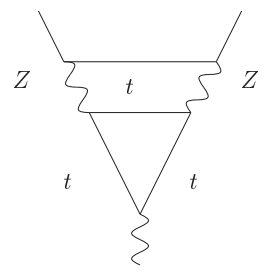

Figure 1. Example of a diagram with a top quark subloop treated with the large top quark mass expansion.

entering the calculation is given in Fig. 1 With $x=M_{Z}^{2} / m_{t}^{2}$, the expansion reads

$$
\begin{aligned}
& \frac{x}{3} \zeta_{2}+\frac{x^{2}}{4}\left(\frac{1}{3} \zeta_{2}-\frac{5}{9}+\frac{1}{3} \log x\right) \\
& +\frac{x^{3}}{5}\left(\frac{1}{9} \zeta_{2}-\frac{79}{240}+\frac{1}{4} \log x\right)+\ldots .
\end{aligned}
$$

Numerically, this gives

$$
0.1483-0.0081-0.0019+0.0003+\ldots .
$$

Such excellent convergence is typical of all neutral current diagrams. The charged current diagrams do not converge so rapidly presumably because of the splitting of the $\mathrm{W}$ boson lines into a topbottom pair as opposed to double top pairs in the previous case.

Let us stress that we performed the expansion exclusively for the vertex diagrams. This means that the propagator diagrams from renormalization, which contain one massive parameter more, the Higgs boson mass, were evaluated by exact one-dimensional integral representations. Our approach is therefore different from that of [10], where expansion was performed in $M_{H}$ as well. In that case, though, only the leading and subleading terms in $m_{t}$ were computed, whereas we obtained the complete result for the fermionic twoloop contributions.

\section{LIGHT FERMION CONTRIBU- TIONS}

The light fermion vertex diagrams contain one scale less and are, therefore, functions of only one 
dimensionless variable. It turned out, that this case allows to perform an exact calculation and obtain the result in closed analytic form expressed through polylogarithms. To this end we used the differential equation method [15].

An example of a scalar integral entering the calculation is given in Fig. 2. Since the subloop is massless it can be integrated to give a massless line with a non-integer power of the denominator. In fact using harmonic tensors it is also possible to perform the full tensor reduction of any scalar integral corresponding to this topology. To get rid of the higher powers of denominators we devised a reduction scheme with the help of integration by parts identities for general indices.

The integral corresponding to Fig. 2] denoted by LF1, satisfies the following differential equation

$M^{2} \frac{d}{d M^{2}} \operatorname{LF} 1(M, m)=$

$\frac{1}{2} \frac{M^{2}}{M^{2}+m^{2}}\left((4-d)\left(4+5 \frac{m^{2}}{M^{2}}\right) \operatorname{LF} 1(M, m)\right.$

$\left.+(10-3 d) \operatorname{LF} 0(M)-(2-d) \frac{1}{m^{2}} \mathrm{~T} 134(0,0, m)\right)$,

where LF0 is the same integral without the massive line, T134 [14] is the sunset vacuum integral, $M^{2}$ is the external momentum squared and $m$ is the mass on the massive line. Both LF1 and LF0 have been made dimensionless with the help of the external momentum.

Equation 4 may be integrated in the class of Nielsen polylogarithms. The polylogarithmic terms of the finite part read

$$
\begin{gathered}
-\mathrm{Li}_{2}(-x)\left(-2+2 \log \left(m^{2}\right)+3 \log (-x)+\log (1+x)\right) \\
+4 \operatorname{Li}_{3}(-x)-\mathrm{S}_{1,2}(-x)+\ldots
\end{gathered}
$$

where $x=M^{2} / m^{2}$. We should note that LF1 has also been calculated in [16].

We were able to integrate analytically all of the integrals but one. The last remaining integral was then evaluated numerically. Recently, another work appeared [17], where several integrals of interest to us have also been calculated. However, the authors of that calculation introduced an extension of the polylogarithm class and

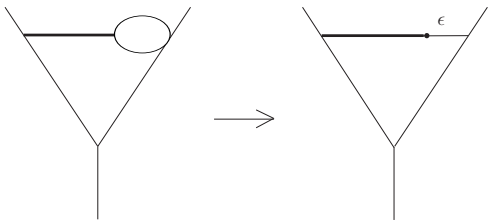

Figure 2. Example of a light fermion subloop integrated using the differential equation technique. The thick line is massive.

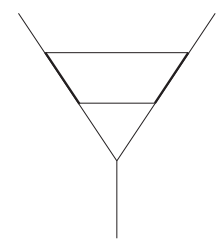

Figure 3. Most complicated light fermion prototype.

represented their result in terms of this extension. In the end, to obtain a numerical result one would need to perform multiple integrals.

Finally, let us note that all integrals have been checked by different expansions in physical and unphysical regimes and by numerical integrations based on dispersion relations [14] and Feynman parameterizations [18]. We have also performed two independent calculations of the onshell renormalization procedure and the necessary counterterms for establishing a finite and meaningful result for $\sin ^{2} \theta_{\text {eff }}^{\text {lept }}$.

\section{DIAGEN/IDSOLVER}

The calculation of the light fermion diagrams described in the last section necessitates a reduction of integrals with irreducible numerators and denominators with higher powers to a small set of master integrals. In the case of the LF1 integral mentioned above, the procedure is relatively simple, since after integration of the subloop it is similar to a one-loop integral. However, in the case of Fig. 3 this is much more difficult. 


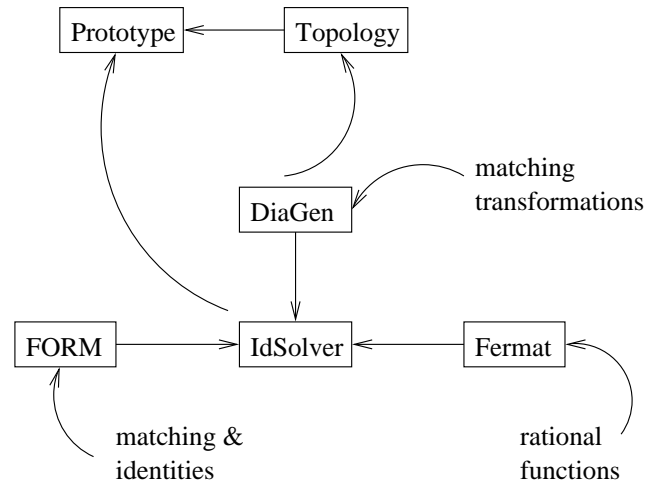

Figure 4. Structure and interrelationships in IdSolver.

To solve this and similar problems, a complete system has been designed and programmed in $\mathrm{C}++$. This system is built upon the DiaGen [13] library which provides tools for diagram generation and topological analysis. The new part, called IdSolver [13], implements, among others, the Laporta algorithm [19]. The major difference compared to the system described in $[19]^{3}$ is the automated topological analysis part.

The general structure and interrelationships with other software used by IdSolver are shown in Fig. 4 Internally, the library is organized around two pairs of classes

\section{- Prototype and PrototypeList}

A prototype is a topology with masses assigned to lines (a mass of an external line is defined through its momentum), i.e. a colored undirected graph in graph theoretical terminology. Identification of isomorphic prototypes is performed either through momentum patterns for vacuum integrals or through topological isomorphism modulo tadpoles. The latter means that the original prototype is decomposed into its subgraph without tadpoles and tadpoles separately, then isomorphism tests are performed on each component. The reason for such sophistication is that (topologically) different

${ }^{3}$ see also [20]. prototypes may be associated with the same integral. The procedure above is unique only for vacuum graphs. However, in all problems considered in practice ${ }^{4}$ no duplicate integrals have been generated. Starting from box graphs, there is a new problem associated with the exchanges of external lines that have the same momentum squared. An algorithm has been implemented which identifies two prototypes as isomorphic only if the necessary interchange does not permute the invariants (e.g. $\mathrm{s}, \mathrm{t}$ and $\mathrm{u}$ for boxes). Besides prototype isomorphism, the Prototype class provides, among others, the topological symmetry group, automatically assigned momenta (with optimization of distribution) and numerator/denominator cancellation rules.

The PrototypeList class manages hierarchies of prototypes. Upon insertion of a prototype it generates all of its subprototypes obtained by canceling lines together with matching rules, i.e. momentum shifting transformations between the prototype and its subprototypes. Simultaneously, it writes administration files to disk starting from declarations needed by FORM [22] and ending with precomputed Integration By Parts (IBP), Lorentz Invariance (if requested by user) or other identities (user defined). Subsequently it manages the solution of the system starting from the simplest prototypes (those that have the least number of lines).

\section{- Integral and IntegralList}

From the point of view of the system, an integral is just a name and list of indices, which represent powers of the irreducible numerators and denominators. Integrals may be equivalent, if they do not have numerators and are equal by symmetry. This property is automatically assigned by the system based on the symmetry group of the prototype. Once solved, integrals have as-

\footnotetext{
${ }^{4}$ this encompasses QED vertices and boxes [21], elec-
} troweak vertices and tadpoles up to four loops. 
sociated expressions, which are linear combinations of other integrals (masters) with rational function coefficients.

The system of integrals is represented by the IntegralList class. The latter provides a method for updating the list with an identity generated by FORM and for writing the solution to disk. It also allows for master integral identification and keeps various statistical information.

The library has several other classes, the description of which we will skip.

The rational function arithmetic needed may be done by any external software by means of a specialized thin interface class. In practice, Maple, Mathematica and Fermat [23] have been used. The latter has proved to be the fastest and most economic in terms of resources required.

An important step in the calculation is the master identification. To be safe, one should solve the system to high powers of numerators and denominators. If the expressions were kept exact, this would be time consuming and unnecessary. To solve this problem, evaluation homomorphisms are used, i.e. the system is solved by projecting the coefficients to the rational numbers field with suitably chosen values of the parameters.

The library DiaGen/IdSolver is a powerful tool many possible applications. It is being currently used to calculate among others the twoloop bosonic corrections to $\sin ^{2} \theta_{\text {eff }}^{\text {lept }}$.

\section{REFERENCES}

1. M. Awramik, M. Czakon, A. Freitas and G. Weiglein, Phys. Rev. D 69 (2004) 053006.

2. M. Awramik and M. Czakon, Phys. Rev. Lett. 89 (2002) 241801, Nucl. Phys. Proc. Suppl. 116 (2003) 238; A. Onishchenko and O. Veretin, Phys. Lett. B 551 (2003) 111; M. Awramik, M. Czakon, A. Onishchenko and O. Veretin, Phys. Rev. D 68 (2003) 053004 .

3. R. Barbieri, M. Beccaria, P. Ciafaloni, G. Curci and A. Vicere, Phys. Lett. B 288 (1992) 95 [Erratum-ibid. B 312 (1993) 511], Nucl. Phys. B 409 (1993) 105; J. Fleischer,
O. V. Tarasov and F. Jegerlehner, Phys. Lett. B 319 (1993) 249, Phys. Rev. D 51 (1995) 3820 .

4. G. Degrassi, P. Gambino and A. Vicini, Phys. Lett. B 383 (1996) 219; G. Degrassi, P. Gambino and A. Sirlin, Phys. Lett. B 394, 188 (1997).

5. A. Freitas, W. Hollik, W. Walter and G. Weiglein, Phys. Lett. B 495 (2000) 338 [Erratumibid. B 570 (2003) 260], Nucl. Phys. B 632 (2002) 189 [Erratum-ibid. B 666 (2003) 305]; M. Awramik and M. Czakon, Phys. Lett. B 568 (2003) 48.

6. A. Sirlin, Phys. Rev. D 22 (1980) 971; W. J. Marciano and A. Sirlin, Phys. Rev. D 22 (1980) 2695 [Erratum-ibid. D 31 (1985) 213].

7. A. Djouadi and C. Verzegnassi, Phys. Lett. B 195 (1987) 265; A. Djouadi, Nuovo Cim. A 100 (1988) 357; B. A. Kniehl, Nucl. Phys. B 347 (1990) 86; F. Halzen and B. A. Kniehl, Nucl. Phys. B 353 (1991) 567; B. A. Kniehl and A. Sirlin, Nucl. Phys. B 371 (1992) 141; B. A. Kniehl and A. Sirlin, Phys. Rev. D 47 (1993) 883; A. Djouadi and P. Gambino, Phys. Rev. D 49 (1994) 3499 [Erratum-ibid. D 53 (1994) 4111].

8. L. Avdeev, J. Fleischer, S. Mikhailov and O. Tarasov, Phys. Lett. B 336 (1994) 560 [Erratum-ibid. B 349 (1994) 597]; K. G. Chetyrkin, J. H. Kühn and M. Steinhauser, Phys. Lett. B 351 (1995) 331; K. G. Chetyrkin, J. H. Kühn and M. Steinhauser, Phys. Rev. Lett. 75 (1995) 3394.

9. M. Faisst, J. H. Kühn, T. Seidensticker and O. Veretin, Nucl. Phys. B 665 (2003) 649.

10. G. Degrassi, P. Gambino and A. Sirlin, Phys. Lett. B 394 (1997) 188.

11. The LEP EWWG and LEP Collaborations, hep-ex/0312023

12. M. Awramik, M. Czakon, A. Freitas and G. Weiglein, hep-ph/0407317.

13. M. Czakon, DiaGen/IdSolver (unpublished).

14. G. Weiglein, R. Scharf and M. Böhm, Nucl. Phys. B 416 (1994) 606; S. Bauberger, F. A. Berends, M. Böhm and M. Buza, Nucl. Phys. B 434 (1995) 383.

15. A. V. Kotikov, Phys. Lett. B 259 (1991) 314; 
E. Remiddi, Nuovo Cim. A 110 (1997) 1435.

16. B. Feucht, J. H. Kühn and S. Moch, Phys. Lett. B 561 (2003) 111.

17. U. Aglietti and R. Bonciani, Nucl. Phys. B 668 (2003) 3; U. Aglietti and R. Bonciani, hep-ph/0401193

18. A. Ghinculov and J. J. van der Bij, Nucl. Phys. B 436 (1995) 30.

19. S. Laporta and E. Remiddi, Phys. Lett. B379 (1996) 283. S. Laporta, Int. J. Mod. Phys. A15 (2000) 5087.

20. Y. Schröder, Nucl. Phys. Proc. Suppl. 116 (2003) 402; C. Anastasiou and A. Lazopoulos, hep-ph/0404258

21. M. Czakon, J. Gluza and T. Riemann, hep-ph/0406203

22. J. A. Vermaseren, math-ph/0010025

23. R. H. Lewis, Fermat, www.bway.net/ lewis/. 